\title{
Primary Clival Mucocele: ENT Perspective in Early Diagnosis and Management
}

\author{
${ }^{1}$ Preetam Chappity, ${ }^{2}$ Alok Thakar
}

\begin{abstract}
Primary clival mucocele is a rare lesion encountered in our daily practice. The previous scant literature enumerate cases which presented with intracranial complications and were usually treated by neurosurgeons. We are presenting a case of a 24-year old female who presented to us with unilateral headache and recurrent episodes of sinusitis. The patient's radiology suggested a primary clival mucocele, which was drained endoscopically. The patient on follow-up is symptom free. This paper tries to implicate the importance of the otorhinolaryngologist in diagnosing this rare lesion and preventing its progression and thus, intracranial complications.
\end{abstract}

Keywords: Clival mucocele, Endoscopic, Treatment.

How to cite this article: Chappity P, Thakar A. Primary Clival Mucocele: ENT Perspective in Early Diagnosis and Management. Clin Rhinol An Int J 2015;8(1):27-29.

Source of support: Nil

Conflict of interest: None

\section{INTRODUCTION}

Mucoceles of paranasal sinuses have never been an alien entity to the ENT surgeon. The patients usually present with significant mucoceles with orbital displacement, facial disfigurement or even intracranial complications. The potency of expansion and probable complications associated with orbit or brain make its early diagnosis and management a vital issue. Usually, the frontoethmoid sinuses are most commonly affected, probably due to their complex drainage, but other sinuses are also involved though less frequently. The sphenoid sinus involved cases usually present to the neurosurgeon with intracranial complications. Cases of primary clival mucoceles are extremely rare, with our search of literature yielding just three case reports of primary clival mucoceles presenting

\footnotetext{
${ }^{1}$ Assistant Professor, ${ }^{2}$ Professor

${ }^{1}$ Department of ENT, All India Institute of Medical Sciences Bhubaneswar, Odisha, India

${ }^{2}$ Department of ENT, All India Institute of Medical Sciences New Delhi, India

Corresponding Author: Preetam Chappity, Assistant Professor, Department of ENT, All India Institute of Medical Sciences, Bhubaneswar, Odisha, India, Phone: 9438884166 e-mail: drcpreetam@aiimsbhubaneswar.edu.in
}

with intracranial complications, like cranial nerve palsies, commonly 6th and 5th, hypopituitarism, etc. They usually warrant a trans-sphenoidal approach for drainage along with long-term antibiotics for improvement. Our case implicates the importance of early diagnosis and endoscopic management of such lesions with minimal associated morbidity and hospital stay.

\section{CASE REPORT}

A 24-year-old female patient presented to us with episodes of recurrent rhinosinusutis since 1 year and left sided generalized headache occasionally since 5 to 6 months. The patient also complained of mild left sided facial heaviness. We treated the patient empirically with oral antibiotics and mucolytic agents, followed by steroid nasal spray. Though the patient had initial good response to treatment, she still complained of persisting symptoms of headache and heaviness. We advised radiology to rule out possible unresolved sinus disease or an impending intracranial complication as the ENT examination including nasal endoscopy did not reveal an obvious pathology. The radiology revealed the presence of minimal ethmoidal sinusitis bilaterally and a left sided clival mucocele with a projection into the posterolateral aspect of the sphenoid sinus. The sphenoid sinus was individually free of disease. The lesion was hyperintense on both T1 and T2 images (Fig. 1). With an informed written consent patient was taken up for endoscopic drainage of the clival mucocele. Intraoperatively, a narrow mouthed pneumatised cell was noticed in the posterolateral wall of sphenoid sinus. The cell was opened and the mouth widened with drainage of a straw colored viscous fluid. The sphenoid sinus mucosa was normal. There was also a $2 \times 2 \mathrm{~mm}$ defect noticed in the posterior wall of the cell with exposed dura (Fig. 2). The absence of cerebrospinal fluid leak was confirmed by positive pressure ventilation and thus, no further repair was done. The patient was discharged on postoperative day 3 after pack removal, with no significant postoperative complications. The histopathological analysis revealed presence of respiratory epithelium with inflammatory cell infiltration. The patient on follow-up is completely symptom free and the repeat radiology after 4 months did not show any further collection. 

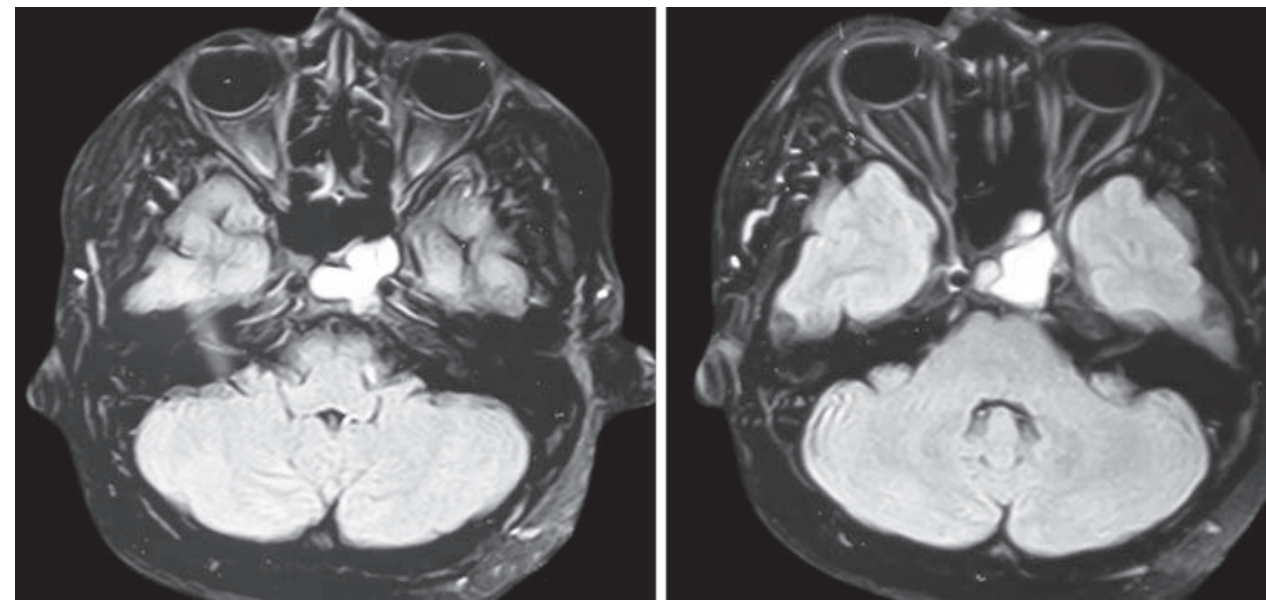

Fig. 1: Extent of the mucocele on MRI scan
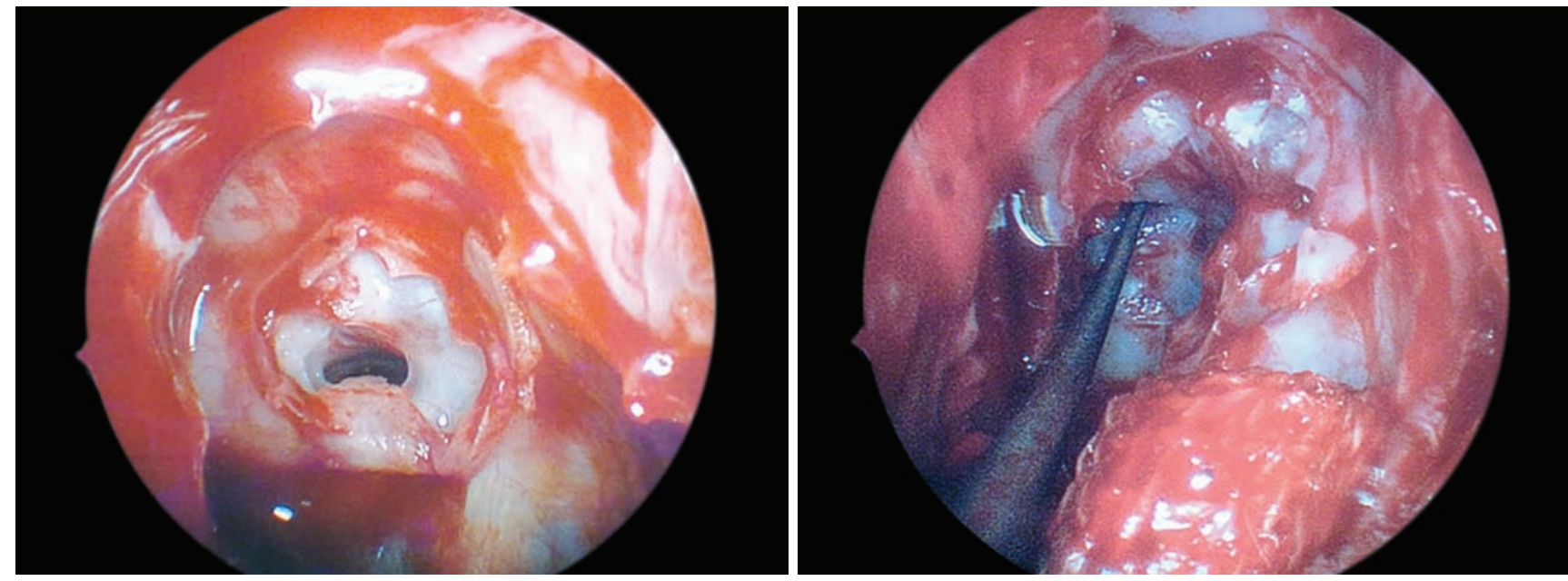

Fig. 2: Post-surgical field after drainage of mucocele

\section{DISCUSSION}

Mucoceles usually have a very long time lag before presentation. In case of post-traumatic or surgical mucocele average period could be about 23 years and in case of an infective etiology the mean time of presentation is 22 months. ${ }^{1}$ The presentation is usually dramatic with a noticeable proptosis, facial swelling or cranial nerve palsies. The exact pathogenesis is not clear as yet, but the obstruction of the sinus ostia associated with inflammation seems the final accepted pathway. The three common pathogenesis theories of importance are, pressure erosion, cystic degeneration of glandular tissue and active bone resorption and regeneration. ${ }^{2}$ The clivus and the dorsam sellae of the sphenoid bone form from the occipital plate in the blastemal skull (desmocranium). The clivus develops to have a compact cortical bone in both anterior and posterior walls, with cancellous bones along with marrow elements in the central position. ${ }^{3}$ As suggested by Fujimoto et al, ${ }^{4}$ the pnuematisation would have progressed in the course of development and formed a communication with the sphenoid sinus which could have become blocked due to the recurrent sphenoid sinusitis associated with blockage of the ostia. Though many a differential diagnosis exist radiology assists us to clinch the diagnosis very well, making it a very essential armatorium in managing the cases. The early diagnosis is of foremost importance and symptoms encountered as in our case, like recurrent sinusitis, with unilateral facial pain and headache, persisting in spite of treatment should raise suspicion. Radiological evaluation is the main mode of diagnosing these cases, with computed tomography (CT) suggesting the cell tracts and magnetic resonance imaging (MRI) guiding us with the extent of lesion and also narrowing our differential diagnosis by exclusion of other pathologies. The endoscopic approach for draining the mucocele seems the gold standard technique at least in early cases. There is a significant decrease in morbidity and hospital stay. Though in our case, the hospital stay extended till 3 days, to rule out a CSF leak. With more experience we could further shorten the hospital stay significantly. We feel the postoperative regular suction cleaning of the cavity is important to prevent a 
recurrence as with other site mucoceles. The high risk of meningitis $(12 \%)$ and death $(19 \%)$ after craniotomy for sphenoidal mucocele extending intracranially certainly skews the balance in favor of endoscopic trans-sphenoidal approach. ${ }^{5}$ The excision of the middle turbinate partially as done by us in our case gives us a better access to sphenoid sinus intraoperatively and also accounts for good postoperative outpatient department (OPD) based cavity cleaning.

\section{REFERENCES}

1. Lund VJ. Anatomical considerations in the etiology of frontoethmoid mucoceles. Rhinology 1987;25:83-88.

2. Lund VJ. Mucoceles. Scott-Brown's Otorhinolaryngology Head and Neck Surgery. 7th ed. 119:1531-1538.

3. Gray's anatomy. 38th ed. Edinburgh, Churchill Livingstone; 1995. p. 271.

4. Fujimoto Y, et al. Primary clival mucocele: case report. Neurol Med Chir (Tokyo); 2011;51:250-252.

5. Nugent GR, Sprinkle P, Bloor BM. Sphenoid sinus mucoceles. J Neurosurg 1970;32:443-451. 